\title{
Prosthetic Rehabilitation of an Amputated finger
}

\author{
Babita Yeshwante ${ }^{1}$, Nikita Parasrampuria ${ }^{2}$,Nazish Baig ${ }^{3}$ \\ ${ }^{I}$ (Department of Prosthodontics and Crown\&Bridge,CSMSS Dental College \& Hospital,Muhs, India) \\ ${ }^{2}$ (Department of Prosthodontics and Crown\&Bridge,CSMSS Dental College \& Hospital,Muhs, India) \\ ${ }_{3}^{3}$ (Department of Prosthodontics and Crown\&Bridge,CSMSS Dental College \& Hospital,Muhs, India)
}

\begin{abstract}
Along with functions like grasping and feeling, hands also have an esthetic impact and they can emphasize the beauty of a gesture or grace of a movement Finger and partial finger amputations are some of the most frequently encountered forms of partial hand loss. Whether the traumatic loss of limb or finger is due to war, congenital malformations, systemic diseases (diabetes), an industrial, domestic or vehicular accident, amputation leaves the individual with a long lasting emotional scar from the disfigurement.The concealment of the amputation with the help of reconstructive surgery or prosthesis usage can shield an amputee from social stigma and help improve emotional healing process. Fabrication of finger prosthesis is as much as an art as it is science. The traditional techniques of prosthesis fabrication as described in the review, via silicone and osseointegrated implant for anchorage of digital prosthesis serves an alternative to complex surgical procedures.
\end{abstract}

Key Words: Amputation, Finger,Implant,Prosthesis,Silicone Osseointegration.

\section{Introduction}

"Amputation" Derived from the Latin word "amputare "(to excise, to cut out) has been defined as the "removal of part or all of a body part enclosed by skin "(online Medical Dictionary). Until the eighteenth century, with the exception of Pare, a few physicians gave thought to the condition of the amputation stump for prosthetic appliance during the period 1846-1847[1].The introduction of antiseptics by Lister allowed surgeons to take more time to produce viable stump capable of supporting prosthetics [2].Amputation causes devastating physical, psychosocial and economic damage to an individual[3].Beasley has noted that individuals who keep their hands hidden inside pockets due to embarrassment over appearance are as functionally disabled as a forequarter ( scapulothoracic) amputee[4].

There are various types of amputations some which are self amputation, congenital amputation, and traumatic amputation which may result from a factory ,farm, powdered tools or motor vehicle accidents ,including industrial or environmental accidents, terrorist attacks and lack of public health which often leads to diabetic, gangrene and /infection. Whatever the indication of an amputation, the result is a limb stump [5].

Finger and partial-finger amputations are some of the most frequently encountered forms of partial hand losses [6].The amount of tissue lost, the current condition of the bone, and involvement of other fingers are some of the factors that have to be considered when choosing a suitable treatment option [7].The primary goal in the treatment of traumatic amputations is to evaluate the suitability of the amputated part of replantation[8].Digital prosthesis fulfills all esthetic requirements, provides some functional gains, restoring movement for the patient and protecting the stump[9].

Several microsurgical techniques such as toe-foot transfer, foot lengthening procedure and use of osteocutaneous flaps may offer opportunities to reconstruct the lost or missing phalanges [10].In case of any contraindications or failure of these surgical methods, esthetic finger prosthesis is an option for the restoration of the handicap [11].

Prosthesis refers to artificial replacement of an absent part of the human body [12].A prosthesis can often restore a "near-normal function" in the distal phalangeal amputation [13]. The finger prosthesis requires an optimum retention for functions such as grasping, carrying and holding [14].

The traditional method of prosthesis is replacing the lost finger by an artificial digit. The artificial digit is made of a silicone elastomer (chemical name- polisiloxane) [15].These silicones can be rendered to match the skin color of the patient and give a more lifelike appearance. Most of silicones used for this purpose are Room temperature vulcanizing silicones (RTV) as they offer chemical inertness, flexibility and elasticity [16].

The second prosthetic mechanism is the use of bone anchored implant retained silicone finger prosthesis as an alternative. Bone anchoring method is used since 1994 as described by Branemark [17].Osseointegrated dental implants have been used to retain prostheses and to avoid the problem of instability. $[10,18,19]$. An implant placed in the intermedullary canal of the residual bone offers additional advantages 
because the technique enables short stumps, where a traditional prosthesis is not successful ,to be restored and provides for tactile sensation by transferring stimuli to the bone through implant[10].

\section{Prosthetic Rehabilitation}

Despite the advances in the microsurgical techniques [20], [21] [22], [23], [24], the reconstruction of the amputated digits for a number of patients may not be successful and the can benefit more with passive prostheses [25], [26]. The primary purpose of prosthesis is to allow the patient to pass unnoticed and concealment of prosthesis usage has been found to be an effective coping strategy.

\subsection{Traditional Prosthetic Finger}

Silicone elastomer of a prosthetic finger is sculpted custom made to suit every individual [15]. Multiple layers of clear silicone over each layer add a life like translucency and protect the coloration from environmental damage [13].

\section{$\checkmark$ FABRication Procedure:}

Most frequently used Glove type prosthesis is described below:

- Impression Making[27]: The patient's hand with missing finger is lubricated with petroleum jelly. The impression material of choice in mostly is irreversible hydrocolloid - alginate. Either the area around the hand can be boxed or a plastic container of sufficient length and diameter (Fig1).

- Model Preparation[27]: The impressions are poured in Type III Dental Stone.

- Wax pattern fabrication : Can be done by 2 methods

1. By Selection of an appropriate donor[28]: The impression of donor's fingers is made with condensation silicone in putty consistency. Molten Wax is poured into the impression. This technique allows the avoidance of laborious task of sculpting.

2. By the impression of the unaffected side [27]- Duplication is done with alginate. (Fig 2).

- Acrylic Nail Preparation and Nail Bed preparation[27]: Tooth colored acrylic resin material is used to fabricate custom made nail. Integral half moons, white margins are incorporated.

- Try in[27]: wax pattern's length and fit is verified on patient's hand(Fig3,4). Artificial nail is removed once the approval from patient is taken.

- Stump model preparation[27,28]: To improve the retention, the circumference of the finger stumps are reduced accurately by $1-1.5 \mathrm{~mm}$ and vertical grooves are created to create vacuum chambers in order to provide a snug fit and vacuum retention.

- Investment Technique[27]: Wax Patterns are invested in typeIII dental stone, using three pour techniques. The mold is dewaxed and separating medium is applied (Fig 5,6).

- Color matching and packing[27]: The silicone (RTV) and pigments are mixed intrinsically to match patient's skin. Color matching for the ventral and dorsal surfaces is done separately in day light. It is packed in the mould (Fig7). Curing is done for 24 hours at room temperature. Prostheses are finished with alpine stones and silicone burs.

- Final prosthesis[27]: to disguise the junction line between the silicone finger prosthesis and residual stump, a wide metal ring or a plastic strip bandage is placed over the margin using a cyanoacrylate adhesive. The final prosthesis is inserted into the residual stump and the fit and color matching is evaluated (Fig8,9). The prosthesis use, maintenance and care instructions are given to the patient.

- Patient's follow up[27]: For prosthesis evaluation the patient is asked to return on Day 1, 7. There after a 6 month follow up is done.

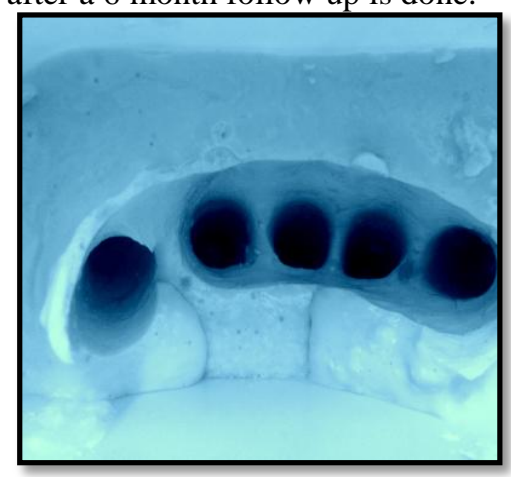

Figure 1: Impression Making

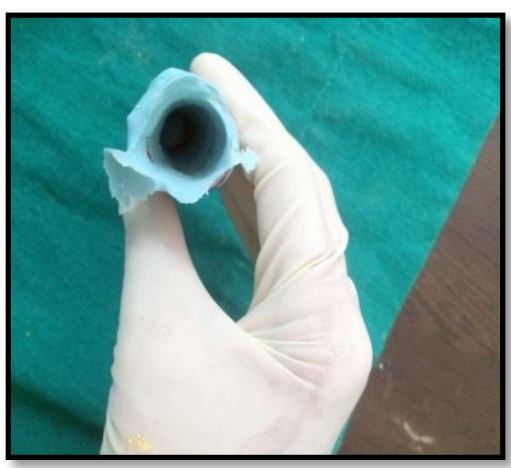

Figure 2:Impression of the finger of unaffected side 


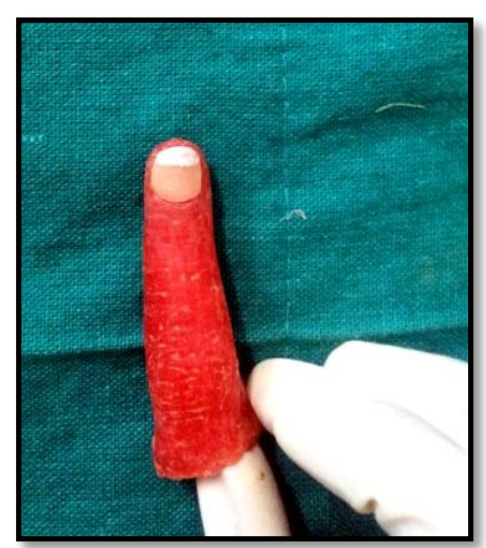

Figure 3:Wax Pattern

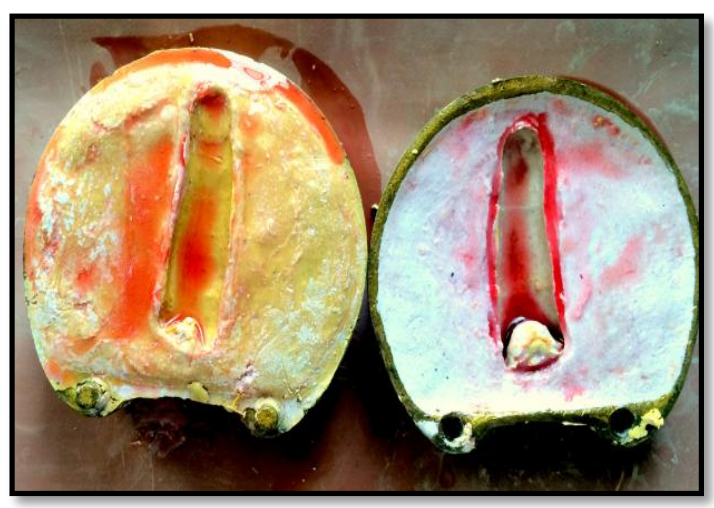

Figure 5: Dewaxing

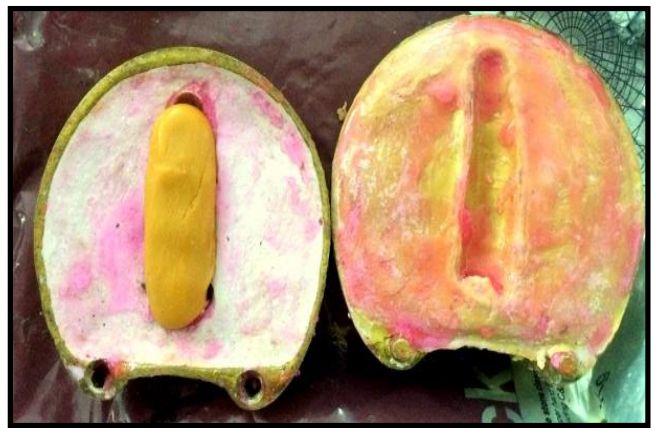

Figure 7: Packing of the colour matched silicone

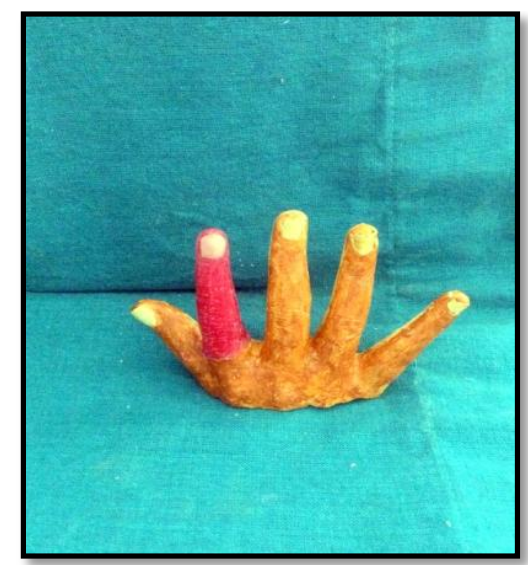

Figure 4: Try in for the wax pattern

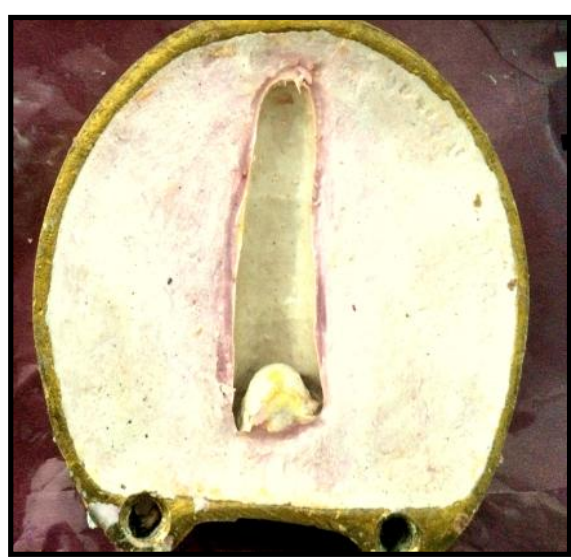

Figure 6: the Mould for packing

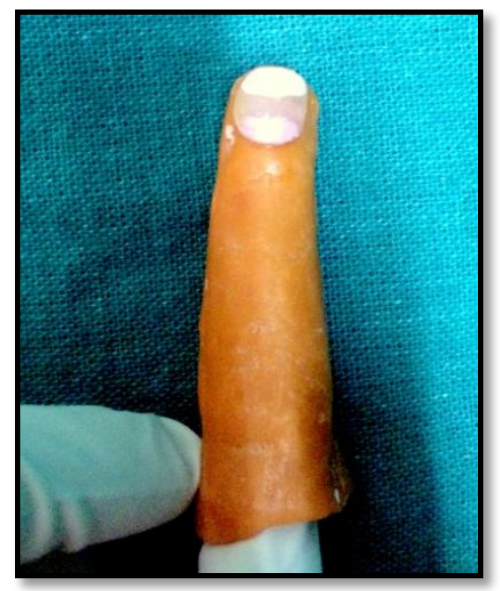

Figure 8: Final Prosthesis 


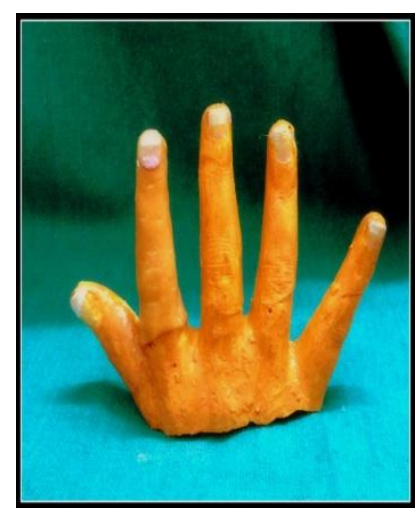

Figure 9: Prosthesis try in

\section{* Exceptional Cases:}

a. If only marginal suction is available for the retention of the prosthesis due to the short or fleshy finger, a medical adhesive may be used to hold the prosthesis in situ [13].

b. Thumb Prosthesis are challenging due to motility and stability requirements [13].

- Interphalangeal amputation: permits suction suspension.

- More extensive loss requires a metacarpal extension and use of a medical adhesive

- A work prosthesis for partial thumb loss may extend to the hand for added stability

- When all or most of the thumb is gone, a pliable wire insert will strengthen the extension and increase the range of possible functions

\section{$>$ Existing Clinical Applications on lines of the Review :}

1. 42 years old male patient whose one right hand finger was partially lost as a result of occupational trauma. A modified impression technique was used for impression making and wax pattern was fabricated using the normal hand. special type of wax was formulated to make a pattern which was easily molded and carved. Two indexed casts were used to determine the length of fingers. Intrinsic and extrinsic staining was also done to match the adjacent skin color. A ring was used as a retentive method[29].

2. 28-year-old man reported with a chief complaint of unaesthetic look due to amputated ring finger of left hand. The remnant stump was missing, the adjacent ring finger was splinted and an adhesive retained prosthesis was planned. The retention was achieved by extending the prosthesis to palmar crease[30].

3. An alternative method of retention by scoring the master cast of a partially amputated finger, thus enhancing the vacuum effect for the retention of the prosthesis[31].

4. Custom-colored prostheses made from silicone elastomers with a simple attachment to make the prosthesis functional to imitate some of the movement[32].

5. For a distal amputation, thimble prosthesis can be used. Besides allowing free range of motion of the proximal finger joints which would otherwise be covered and restricted with fitting finger prosthesis, it also minimizes skin coverage for optimal sensibility and is easier to don and doff, requires5-7\% circumference reduction[33].

6. Socket of finger prosthesis reduced by $2 \mathrm{~mm}$ less than the original value, and a central tunnel with 4 $\mathrm{mm}$ width and depth with length matching the distance between the end of the stump and the nail section was provided, so that the silicone material extended to the stump to provide appreciable suspension[34].

7. A plastic cylindrical small open container (impression cap) was used for making an impression of the fingers. A wax pattern of the missing part was made by replicating the same fingers of the other hand[35]. 
Hence, the overall durability and stain resistance of silicone is far superior to any other material currently available for finger restorations. Almost all stains, including the ball point ink, newsprint, clothing dyes, and food colorings can be removed easily with soap and water [13].The gentle constant pressure of an elastomer prosthesis helps to desensitize and protect the injured tip. [36]. Recent literature speculates that silicone gel improves the hydration of stratum corneum of immature hypertrophic scars [37].

But this poor adhesive ability limits the force that the prosthesis could withstand before detachment. Thus pure silicone elastomer prosthetic finger has mainly cosmetic purposes and low functionality [15].

\subsection{Implant Retained Finger Prosthesis}

The second prosthetic mechanism used to overcome the problems of simple silicone prosthesis. A metal piece is inserted and then implanted into the terminal bone of the amputation which is called osseointegration [15].

In 1952, Prof Per-Ingvar Branemark of Sweden used a titanium implant chamber to study blood flow in rabbit bone. At the end of the experiment he discovered that the bone had integrated so completely with the implant that the chamber could not be removed. Branemark called this discovery "osseointegration" [38].The discovery of metal integrating into the bone was then used in other medical fields, such as dentistry and maxillofacial reconstruction [8].

\section{Existing Clinical Applications on lines of the Review:}

1. A 30-year-old male whose fourth and fifth fingers of his right hand were lost at the level of the distal interphalangeal joint as a result of an accident. The first stage of surgical procedure was to raise an ellipsoid flap on the stumps under local anesthesia. Drill holes were prepared longitudinally in appropriate dimensions for the implants, in the medullar canals of the middle phalanxes of both digits. Two dental implant with the dimensions of $4.6 \mathrm{~mm} \times 12 \mathrm{~mm}$ and $3.8 \mathrm{~mm} \times 10 \mathrm{~mm}$ were placed in the prepared holes respectively. At the second surgical operation, the skin was reopened, cover screws on the implants were removed, and healing caps were attached to implant fixtures. At the end of 15-day healing period, impressions were taken using silicone impression material after attaching impression caps (close tray) in place. Once the impression material had set, implant analogues were attached to the impression caps that were removed from the fingers[39].

2. The use of osseointegrated implants for retention of finger prostheses in a patient with amputated thumb and index finger. Dental implants were placed in the residual bone of the fingers using two-stage surgery. Custom-made attachments were used to provide retention between implants and silicone prostheses. Prosthetic fingernails were made of composite resin material. After 6 months, implants were found to be clinically successful. The complications of broken prosthetic nail and mild discoloration were observed[40].

3. The implant retained finger prosthesis was chosen by using one stage surgery technique. Then, Right Branchial nerve was anaesthetized and hemostasis was obtained using the tourniquet. Skin incision was given at the implant site; the position and the angulations of the implant were guided by the fluoroscopy radiation. Then implant of $5 \mathrm{~mm}$ diameter X $17 \mathrm{~mm}$ length was placed in the bone inside the medullary canal with the insertion torque. Radiograph was taken to verify the position of the implant[41].

4. The $\mathrm{O}$ ring retention system was used with a modified hexagon -shaped base and a metallic capsule adapted to the acrylic resin to attach the prosthesis to the implant[42].

5. The titanium fixture was allowed to osseointegrate with the phalanx of bone, a skin penetrating titan magnetic abutment was placed on the top of the fixture, to which the prosthesis is firmly attached[19].

6. Direct skeletal fixation of limb prostheses using an intraosseous transcutaneous implant which is carried out in 2 operations. The implant may be in one piece or modular with a separate abutment. The surface may be modified (for e.g. by means of screw thread, porous or roughened surface or addition of a special coating) to enhance bone and soft tissue integration[43].

7. To evaluate stress distributions in finger bone when the loading force is applied along the long axis of the implant using finite element analysis. The fingerbone model containing cortical bone and cancellous bone was constructed by using radiograph. Astra Tech Osseo Speed bone level implant of 4.5 X $14 \mathrm{~mm}$ 
length was selected. The force was applied to the top of the abutment along the long axis of the implant. The maximum stress was located at the head of abutment screw. The minimum stress was located in the apical third of the implant fixture[44].

The Alternate connection and substructure designs for implant retained prosthesis have been described below [15]:

Table 1:

\begin{tabular}{|c|c|}
\hline $\begin{array}{l}\text { ALTERNATE CONNECTION DESIGN[15] } \\
\text { (connect the terminal bone to the prosthesis) }\end{array}$ & $\begin{array}{l}\text { ALTERNATE SUBSTRUCTURE DESIGN[15] } \\
\text { (The purpose of the substructure is to regain some functionality and } \\
\text { motility of the finger.) }\end{array}$ \\
\hline $\begin{array}{l}\text { 1. Screw and clip :functions with the installation of a spring } \\
\text { loaded shaft in the terminal end of osseointegrated } \\
\text { abutment with peripheral clip wells. }\end{array}$ & $\begin{array}{l}\text { Spring-Loaded Sac } \text { - Describes the connection between two } \\
\text { solid bone-like segments. }\end{array}$ \\
\hline $\begin{array}{l}\text { 2. Magnet and clip : The prosthesis magnetic terminal end is } \\
\text { aligned and attached to the oppositely magnetized well in } \\
\text { the abutment. }\end{array}$ & $\begin{array}{l}\text { 2. Mechanical Joint with spring - This design describes a round } \\
\text { joint casing attached to the unmovable portion of the finger } \\
\text { prosthetic. }\end{array}$ \\
\hline $\begin{array}{l}\text { 3. Allen Wrench : Functions with the installation of a simple } \\
\text { titanium abutment that extends beyond the length of the } \\
\text { terminal bone and is fitted with a slot. }\end{array}$ & $\begin{array}{l}\text { Flat Piece - Consists of a sturdy, flat piece of metal or dental } \\
\text { acrylic that is firmly connected to the implanted abutment and } \\
\text { bent at a natural angle of a finger at rest. }\end{array}$ \\
\hline $\begin{array}{l}\text { 4. Reverse Screw and clip : Installation of the titanium } \\
\text { abutment that extends beyond the length of terminal bone } \\
\text { with a flared conical tip. }\end{array}$ & 4. $\frac{\text { Articulation Mechanism- }}{\text { undergo active flexion }}$ Consists of movable parts that \\
\hline
\end{tabular}

Hence the implant retained finger prosthesis offers several benefits over conventional prosthesis [38].

- Less feeling of weight

- More control over prosthesis

- No perspiration, pain and tissue breakdown from the socket

- Easier donning and doffing

- Osseoperception - partial recovery of tactile sensation by transferring stimuli to the bone through implant because of direct pressure of implant on the bone. research suggests that osseoperception is secondary to nerve ingrowth into remodeling bone, as controlled by neuropeptides such as calcitonin gene related peptide

- Current disadvantages of osseointegration are[38]:

- Two surgeries are required

- Relatively long rehabilitation period

- Deep infection risk

\section{Inference}

The goals of rehabilitation: preserve the functional length, useful sensitivity, prevent symptomatic neuromas, adjacent joint contractures, achieve short duration morbidity, and enable the patient to perform tasks of daily life as quickly as possible [45].

Buckner $\mathrm{H}$ et al stated that the acceptance rate of individually sculpted custom made silicone prosthesis has been much higher [46].

As well as excellent cosmetic result, osseointergrated implant restorations provide several advantages such as stable fixation of the prosthesis to the skeleton and restoration of some sensory feedback (osseoperception) [47]. Retention in finger prosthesis is generally achieved by a vacuum effect on the stump [48], use of medical adhesives [49] and placement of finger ring [8]. Michael j.w. et al advocated the use of separate vacuum chambers formed from the biostar clear acrylic of $1.5 \mathrm{~mm}$ thickness to get the suction and enhance retention [13] Ten consecutive patients performed a battery of hand function tests and rated their ability to perform a variety of activities of daily living both with and without their prosthesis using the Disabilities of the Arm, Shoulder, and Hand questionnaire [50].

Results show a significant improvement in 3-finger-pinch strength and grip strength and a trend of improvement of tip-pinch, lateral-pinch, and grip strength in dynamometer positions [50]. 


\section{Conclusion}

With the advent of revolutionary advancements the prosthetic rehabilitation of an amputated finger has become less challenging and offers several cosmetic and functional advantages over the complex surgeries. Both the traditional silicone prosthesis and implant retained finger prosthesis offer good retention and stability and aids to restore the near normal function of the finger.

To summarize, the only path to successful rehabilitation is the one which relieves the patient off the social stigma imposed onto him by the society.

\section{REFERENCES}

[1]. N. Shanmuganathan, M. Uma Maheswari, and Ahmed Hasan Jibran,Aethetic Finger Prosthesis

[2]. Meier RH (2010) History of arm amputation, prosthetic restoration, and arm amputation rehabilitation. http://www.demosmedpub.com/files/Meier_01.pdf

[3]. Parkes CM. Psycho-social transitions: Comparison between reactions to loss of a limb and loss of a spouse. British Journal of Psychiatry. 1975; 127:204-210. [PubMed]

[4]. Beasley (1981)Op cit.744

[5]. Fassler PR. Fingertip injuries: evaluation and treatment. J Am Acad Orthop Surg.1996; 4(1):84-92. [PubMed]

[6]. Pillet J. The Aesthetic hand prosthesis. Orthop Clinics in North Amer 1981;12;961-70

[7]. Aydin C, Karakoca S, Yilmaz H. Implant-retained digital prostheses with custom-designed attachments: A clinical report. Journal of Prosthetic Dentistry. 2007; 97: 191-95.

[8]. Boulas HJ Amputations of the fingers and Hand: Indications for replantation. J.Am.Acad.Ortho.Surg, Mar 1998; 6:100-105

[9]. Sierakowski A1, Watts C, Thomas K, Elliot D. .Long-term outcomes of osseointegrated digital prostheses for proximal amputation. J Hand Surg Euro Vol. 2011 Feb; 36(2):116-25. Epub 2010 Aug 31

[10]. Doppen P, Solomons M, Kritzinger S. Osseointegrated finger prostheses. Journal of Hand Surgery European. $2009 ; 34: 29-34$.

[11]. Cervelli V, Bottini DJ, Arpino A, Grimaldi M, Rogliani M, Gentile P. Bone anchored implant in cosmetic finger reconstruction. Annales de chirurgie plastique esthetique.2008; 53:365-36.anplas.2007.06.010. [PubMed]

[12]. GPT,J Prosthodent,2005;94910:10-92

[13]. JohnW.Michael,MEd,CPO ,Horst Buckner, MDT, CDT : Options for Finger Prostheses

[14]. Pereira BP, Kour AK, Leow EL, Pho RWH (1996) Benefits and use of digital prostheses. Journal of Hand Surgery 21: $222-228$.

[15]. Gregory Cion,Medical Art Prosthetics,LLC, Implant Retained Finger Prosthesis

[16]. Kanter JC.The Use of RTV silicones in maxillofacial prosthetics.J Prosthet Dent 1970;24960:646-53

[17]. Lundborg G, Branemark PI, Rosen B. Osseointegrated thumb prostheses: a concept for fixation of digit prosthetic devices. J Hand Surg [Am]. 1996; 21(2): 216-21

[18]. Manurangsee P, Isariyawut C, Chatuthong V et al. (2000) Osseointegrated finger Prosthesis: an alternative method for finger reconstruction. The Journal of Hand Surgery 25A: $86-92$.

[19]. Infanger M. [Improved fixation of finger prostheses by means of intramedullary titanium anchors and magnets

[20]. Bickel KD, Dosanjh A. Fingertip reconstruction. Journal of Hand Surgery. 2008; 33:1417-1419. [PubMed]

[21]. Hattori Y, Doi K, Sakamoto S, Yamasaki H, Wahegaonkar A, et al. Fingertip replantation. Journal of Hand Surgery. 2007; 32:548555. [PubMed]

[22]. Shi D, Qi J, Li D, Zhu L, Jin W, et al. Fingertip replantation at or beyond the nail base in children. Microsurgery. 2010; 30:380385. [PubMed]

[23]. Beris AE, Lykissas MG, Korompilias AV, Mitsionis GI, Vekris MD, et al. Digit and hand replantation. Archives of Orthopaedic and Trauma Surgery. 2010;130:1141-1147.[PubMed]

[24]. Molski M. Replantation of fingers and hands after avulsion and crush injuries. Journal of Plastic, Reconstructive and Aesthetic Surgery. 2007; 60:748-754. [PubMed]

[25]. Alison A, Mackinnon SE. Evaluation of digital prostheses. Journal of Hand Surgery.1992; 17:923-926. [PubMed

[26]. Wilson RL, Carter Wilson MS. Rehabilitation after amputations in the hand. Orthopedic Clinics of North America. 1983; 14:851872. [PubMed]

[27]. Vikas B. KambleA, Raviraj G. DesaiB, Kashinath C. ArabbiC, Kaustubh MahajanD, Siddharam PatilD: Finger Prostheses for Multiple Finger Amputations: Two Case Reports".

[28]. Dhruv Arora, Shyam Singh, R Shakila, SK Jagdish, Santosh Anand, VR Arun Kumar, J Balaji: Finger Prostheses - Overcoming a Social Stigma: Clinical Case Reports.

[29]. Kumar L, Saloni, Rao J, Mattoo KA, Yadav: A Finger prosthesis with an alternative approach: Prosthet Orthot Int. 2013 Apr;37(2) Epub 2012 Jul 24.

[30]. Goyal A1, Goel H,Prosthetic rehabilitation of a patient with finger amputation using silicone material Prosthet Orthot Int. 2014 Apr 1. [Epub ahead of print]

[31]. Jacob PC1, Shetty KH, Garg A, Pal B.Silicone finger prosthesis. A clinical report.:J Prosthodont. 2012 Dec;21(8):631-3

[32]. Pattanaik B, Pattanaik S .Fabrication of functional finger prosthesis with simple attachment:J Indian Prosthodont Soc. 2013 Dec;13(4):631-4. Epub 2012 Dec 8

[33]. Leow ME1, Pereira BP, Kour AK, Pho RW.Aesthetic life-like finger and hand prostheses: prosthetic prescription and factors influencing choice: Ann 1997 Nov;26(6):834-9.

[34]. Arazpour M1, Mardani MA, Ahmadi Bani M, Zarezadeh F, Hutchins SW, Design and fabrication of a finger prosthesis based on a new method of suspension

[35]. Tripathi S1, Singh RD, Chand P, Mishra N, Yadav LK, Singh SV. A modified approach of impression technique for fabrication of finger prostheses.

[36]. Livingstone DP. The D-Z stump protector. Am J Occup Ther. 1988;42:185-187.[PubMed]

[37]. Burkhardt A, Weitz J. Oncologic applications for silicone gel sheets in soft-tissue contractures. Am J Occup Ther. 1990;45(5):460462. [PubMed]

[38]. Miki Fairley, Osseointegration: In the wave of future ?

[39]. Aydin Ozkan, Bugra Senel, Can Engin Durmaz, Hasan Alper Uyar, Rahmi Evinc :Use of Dental Implants to Retain Finger Prostheses: A Case Report. 
[40]. Aydin C, Nemli SK,Yilmaz H. Esthetic, functional, and prosthetic outcomes with implant-retained finger prostheses. Prosthet Orthot Int. 2013 Apr;37(2):168-74. Epub 2012 Jul 24.

[41]. Pokpong Amornvit, Dinesh Rokaya, Konrawee Keawcharoen, Somchart Raucharernporn and Nimit Thongpulsawasdi ,One- Vs Two Stage Surgery Technique for Implant Placement in Finger Prosthesis, Journal of Clinical and Diagnostic Research. 2013 Sept, Vol-7(9): 1956-1958

[42]. Goiato MC1, dos Santos DM, Amoroso AP, Gennari Filho H, Dekon SF :Implant-retained finger prosthesis with modified retention system,

[43]. NICE interventional procedure guidance 270,www.nice.org.uk/ipg270

[44]. PokpongAmornvit, Dinesh Rokaya, Konrawee Keawcharoen and Nimit Thongpulsawasdi Stress Distribution in Implant RetainedFinger Prosthesis: A Finite Element Study :Journal of Clinical and Diagnostic Research. 2013 Dec, Vol-7(12): 2851-28

[45]. Ware LC, et al. Digital amputation and ray resection. In: Clark GL, et al., editors. Hand rehabilitation: a practical guide. 2. New York: Churchill Livingstone, Inc; 1998

[46]. Buckner H.Cosmetic hand prosthesis-a case report. Orthot Prosthet.1980; 34(3):41-45

[47]. Brnemark R, Brnemark, P-I, Rydevik B, Myers RR. Osseointegration in skeletal reconstruction and rehabilitation: A review. Journal of Rehabilitation Research and Development. 2001; 38: 175-181.

[48]. Jean Pillet, Evelyn J. Mackin.O and P Library Aesthetic Restoration. Atlas of Limb Prosthetics: Surgical, Prosthetic, and Rehabilitation Principles: Partial-Hand Amputations.

[49]. Pillet J. Esthetic hand prostheses. J Hand Surg [Am] 1983; 8:778-81

[50]. Lifchez SD1, Marchant-Hanson J, Matloub HS, Sanger JR, Dzwierzynski WW, Nguyen HH Functional improvement with digital prosthesis use after multiple digit amputations.,J Hand Surg Am. 2005 Jul; 30(4):790-4.

\section{FIGURE REFERENCES}

Fig 1: CSMSS Dental College and Hospital, Nikita Parasrampuria, First Year MDS PG Fig 2: CSMSS Dental College and Hospital, Nikita Parasrampuria, First Year MDS PG Fig 3: CSMSS Dental College and Hospital, Nikita Parasrampuria, First Year MDS PG Fig 4: CSMSS Dental College and Hospital, Nikita Parasrampuria, First Year MDS PG Fig 5, 6: CSMSS Dental College and Hospital, Nikita Parasrampuria, First Year MDS PG Fig 7: CSMSS Dental College and Hospital, Nikita Parasrampuria, First Year MDS PG Fig 8: CSMSS Dental College and Hospital, Nikita Parasrampuria, First Year MDS PG Fig 9: CSMSS Dental College and Hospital, Nikita Parasrampuria, First Year MDS PG 